\title{
Mixing zones in magnetized differentially rotating stars
}

\author{
V. Urpin ${ }^{1,2}$ \\ 1 Departament de Fisica Aplicada, Universitat d'Alacant, Ap. Correus 99, 03080 Alacant, Spain \\ e-mail: vadim.urpin@uv.es \\ 2 A. F. Ioffe Institute of Physics and Technology and the Isaac Newton Institute of Chili, Branch in St. Petersburg, 194021 St. Petersburg, \\ Russia
}

Received 5 April 2005 / Accepted 28 September 2005

\section{ABSTRACT}

We study the secular instability of magnetized differentially rotating radiative zones taking account of viscosity and magnetic and thermal diffusivities. This considered instability generalizes the well-known Goldreich-Schubert-Fricke instability for the case of a sufficiently strong magnetic field. In magnetized stars, instability can lead to formation of non-spherical, unstable zones where weak turbulence mixes the material between the surface and interiors. Such unstable zones can manifest themselves by a non-spherical distribution of elements on the stellar surface.

Key words. magnetohydrodynamics (MHD) - instabilities - magnetic fields - turbulence

\section{Introduction}

The effect of the magnetic field on stellar differential rotation was considered by many authors. In his pioneering work, Fricke (1969) first pointed out the importance of thermal diffusion for stability of magnetized differentially rotating stars. This conclusion was developed further in the paper by Acheson (1978), who consistently took the main diffusive processes (viscosity, thermal and magnetic diffusion) into account and noted that even a weak kinetic effect such as viscosity can influence the secular stability of magnetized stellar radiative zones. Acheson (1978) reviewed in detail the so called double diffusive instabilities that can occur in stellar radiative zones because of dissipative effects. Perhaps the diffusive instability the best known in an astrophysical context is one that is considered by Goldreich \& Schubert (1967) and Fricke (1968). In non-magnetic stars, this instability arises if the angular velocity depends on the height above the equatorial plane and it can be responsible, for example, for the mixing and angular momentum transport in stellar radiative zones (see, e.g., Knobloch \& Spruit 1982; Korycansky 1991). Note that some double diffusive instabilities can be important for the rotational evolution of degenerate stars as well (Urpin 2003a,b).

Most likely, however, is that the magnetic field may exist in stellar radiative zones despite many uncertainties regarding the origin, evolution, and geometry of these fields. It seems that even magnetic buoyancy cannot substantially reduce the field in radiative zones because of the stabilizing influence of stellar rotation on magnetic buoyancy instability. Generally, rotation can suppress this instability if the rotation velocity, $V$, is larger than the Alfvén velocity, $V>c_{\mathrm{A}}$ (see, e.g., Gilman 1970; Acheson \& Gibson 1978; Acheson 1979).

Secular instabilities are typically sensitive to the magnetic field, and the stability properties of magnetic and non-magnetic secular modes can be different. The influence of the toroidal magnetic field on the Goldreich-Schubert-Fricke instability has been considered by Acheson (1978), who noted that this sort of field plays a destabilizing role and can qualitatively change the criterion of rotational instability. This effect can be important, for example, for the sun where the toroidal field is expected to be buried deep inside the radiative zone (Schmitt \& Rosner 1983). Note that the destabilizing influence of the toroidal field is not surprising because such a field leads to instability even in non-rotating stars (see Tayler 1973; Goossens \& Tayler 1980).

The effect of the poloidal field is more complicated and, to the best of our knowledge, has not been considered in its full generality. Recently, Menou et al. (2004) considered diffusive instabilities that occur in differentially rotating magnetized radiative zones and obtained the criteria of instability in the limit when either viscosity or magnetic diffusivity is vanishing. This consideration, however, is restricted by the case of a very weak magnetic field. The authors find that stability properties are sensitive to the combination of viscosity, magnetic, and thermal diffusivities and, following Acheson (1978), conclude that all three should be included in the analysis from the very beginning. The results of this consideration have been applied by Menou (2004) to account for millisecond oscillations in differentially rotating atmospheres of accreting weakly-magnetized neutron stars. 
The present paper extends the study of secular instability to the case of a relatively strong magnetic field and arbitrary diffusivities. We show that such the magnetic field changes the stability properties of radiative zones drastically. In a strong field, the instability of differential rotation can lead to formation of non-spherical unstable zones where weak turbulence is generated. The geometry of these zones depends on the magnetic configuration and rotation. For example, in the simplest case of an axisymmetric field, the turbulent zones are formed near the rotation axis and around the equator. If the field is not axisymmetric then the instability will lead to the formation of cone-like unstable zones within the radiative region. Turbulent motions mix the material in the unstable zones, so a fraction of the material from deep interiors may reach the surface where chemically peculiar regions should be formed.

An outline of the paper is as follows. In Sect. 2 we derive the general dispersion equation taking account of viscosity and of the magnetic and thermal diffusivities. The criteria of instability are discussed in Sect. 3, and the growth time of secular instability is considered in Sect. 4. A discussion of results follows in Sect. 5.

\section{Basic equations and the dispersion relation}

The MHD equations governing the velocity, magnetic field and thermal balance in the stellar radiative zone are

$$
\begin{aligned}
\dot{\boldsymbol{v}}+(\boldsymbol{v} \cdot \nabla) \boldsymbol{v}= & -\frac{\nabla p}{\rho}+\boldsymbol{g}+\frac{1}{4 \pi \rho}(\nabla \times \boldsymbol{B}) \times \boldsymbol{B} \\
& +v\left[\Delta \boldsymbol{v}+\frac{1}{3} \nabla(\nabla \cdot \boldsymbol{v})\right],
\end{aligned}
$$

$\dot{\rho}+\nabla \cdot(\rho \boldsymbol{v})=0$,

$\dot{\boldsymbol{B}}-\nabla \times(\boldsymbol{v} \times \boldsymbol{B})=-\nabla \times[\eta \cdot(\nabla \times \boldsymbol{B})]$,

$\nabla \cdot \boldsymbol{B}=0$,

$\rho c_{p} \dot{T}-\beta \dot{p}+\rho c_{p} v \cdot(\Delta \nabla T)=\nabla \cdot(\kappa \cdot \nabla T)$

(see, e.g., Landau \& Lifshitz 1981). Our notation is as follows: $\boldsymbol{v}$ is the fluid velocity; $\rho, p, T$ are the density, pressure and temperature, respectively; $\boldsymbol{B}$ is the magnetic field; $\boldsymbol{g}$ gravity; $\Delta \nabla T=\nabla T-\nabla_{\mathrm{ad}} T$ is a difference between the real and adiabatic temperature gradients; $\nabla_{\mathrm{ad}} T=\beta \nabla p / \rho c_{p} ; \beta=-(\partial \ln \rho / \partial \ln T)_{p}$; $c_{p}$ is the specific heat at constant pressure; $v$ is the kinematic viscosity; and $\eta$ and $\kappa$ are the magnetic diffusivity and thermal conductivity, respectively. We ignore the spatial dependence of the kinetic coefficients $v, \eta$, and $\kappa$, which is appropriate for short wavelength analysis.

We work in cylindrical coordinates $(s . \varphi, z)$ with the unit vectors $\left(\boldsymbol{e}_{s}, \boldsymbol{e}_{\varphi}, \boldsymbol{e}_{z}\right)$ and assume that the fluid rotates with the angular velocity $\Omega=\Omega(s, z)$ in the unperturbed state. We assume that the Alfvén speed, $c_{\mathrm{A}}=B / \sqrt{4 \pi \rho}$, is small compared to the sound speed, $c_{\mathrm{s}}$. Then, the magnetic field should satisfy the condition

$B<3 \times 10^{7} \sqrt{\rho T_{6}}$ G,

where $\rho$ is measured in $\mathrm{g} / \mathrm{cc}$, and $T_{6}=T / 10^{6} \mathrm{~K}$. This assumption still leaves a wide range of $B$ to appreciably influence the stability properties, and they are of interest for stellar radiative zones. Note that under the condition $c_{\mathrm{S}}>c_{\mathrm{A}}$, the Alfvén speed can be larger than the rotation velocity $s \Omega$.

In the unperturbed state, the star is assumed to be in hydrostatic equilibrium in the $s$ - and $z$-directions,

$$
\frac{\nabla p}{\rho}=\boldsymbol{G}+\boldsymbol{G}_{B}, \quad \boldsymbol{G}=\boldsymbol{g}+\Omega^{2} \boldsymbol{s}, \boldsymbol{G}_{B}=\frac{(\nabla \times \boldsymbol{B})}{4 \pi \rho} \times \boldsymbol{B} .
$$

Generally, even if $c_{\mathrm{s}}>c_{\mathrm{A}}$ and the unperturbed Lorentz force is small compared to the pressure force, the effect of the magnetic field cannot be neglected in this equation. Taking the curl of Eq. (7), we obtain

$\frac{1}{\rho^{2}} \nabla p \times \nabla \rho=\boldsymbol{e}_{\varphi} s \frac{\partial \Omega^{2}}{\partial z}+\boldsymbol{L}$,

where

$\boldsymbol{L}=\frac{1}{4 \pi} \nabla \times\left[\frac{1}{\rho}(\nabla \times \boldsymbol{B}) \times \boldsymbol{B}\right]$.

Therefore, the magnetic field (as well as rotation) can be responsible for the baroclinic effect that is oftenly crucial for stability. The Lorentz force splits into potentional (magnetic pressure) and solenoidal (magnetic tension) parts. In our study, the effect of the both parts on the unperturbed state is consistently taken into account.

Consider stability of axisymmetric short wavelength perturbations with the spacetime dependence $\propto \exp (\gamma t-i \boldsymbol{k} \cdot \boldsymbol{r})$, where $\boldsymbol{k}=\left(k_{s}, 0, k_{z}\right)$ is the wavevector, $|\boldsymbol{k} \cdot \boldsymbol{r}| \gg 1$. Small perturbations will be indicated by subscript 1 , while unperturbed quantities will have no subscript, except for indicating vector components. In the local approximation, the linearized MHD-equations read

$$
\begin{array}{r}
\left(\gamma+\omega_{v}\right) \boldsymbol{v}_{1}+\frac{v}{3} \boldsymbol{k}\left(\boldsymbol{k} \cdot \boldsymbol{v}_{1}\right)+2 \boldsymbol{\Omega} \times \boldsymbol{v}_{1}+s \boldsymbol{e}_{\varphi}\left(\boldsymbol{v}_{1} \cdot \nabla \Omega\right)= \\
\mathrm{i} k \frac{p_{1}}{\rho}+\boldsymbol{G} \frac{\rho_{1}}{\rho}-\frac{\mathrm{i}}{4 \pi \rho}\left(\boldsymbol{k} \times \boldsymbol{B}_{1}\right) \times \boldsymbol{B},
\end{array}
$$

$\gamma \rho_{1}+\rho \boldsymbol{k} \cdot \boldsymbol{v}_{1}=0$,

$\left(\gamma+\omega_{\eta}\right) \boldsymbol{B}_{1}-\boldsymbol{s} \boldsymbol{e}_{\varphi}\left(\boldsymbol{B}_{1} \cdot \nabla \Omega\right)=-i(\boldsymbol{k} \cdot \boldsymbol{B}) \boldsymbol{v}_{1}$

$\boldsymbol{k} \cdot \boldsymbol{B}_{1}=0$,

$\left(\gamma+\omega_{\chi}\right) T_{1}-\frac{\gamma \beta}{\rho c_{p}} p_{1}=-v_{1} \cdot(\Delta \nabla T)$,

where the characteristic inverse timescales of the viscous and ohmic dissipation and the thermal diffusion are given by $\omega_{\nu}=\nu k^{2}, \omega_{\eta}=\eta k^{2}$ and $\omega_{\chi}=\chi k^{2}$, respectively; $\chi=\kappa / \rho c_{p}$ is the thermal diffusivity.

Perturbations of the pressure in Eq. (9) can be expressed in terms of perturbations of the density and temperature, using the equation of state,

$\frac{p_{1}}{p}=\left(\frac{\partial \ln p}{\partial \ln \rho}\right)_{T}\left(\frac{\rho_{1}}{\rho}+\beta \frac{T_{1}}{T}\right)$. 
We consider the dispersion relation in the case $k c_{\mathrm{s}} \gg \gamma$ which corresponds to the Boussinesq approximation for a non-magnetic fluid. Apart from this, the applicability of the Boussinesq approximation in the magnetic field imposes a restriction on the field strength. Namely, the magnetic field should satisfy the inequality $c_{\mathrm{A}}^{2} / c_{\mathrm{s}}^{2} \ll(k H)^{-1}$, where $H$ is the lengthscale of unperturbed quantities. Solving Eqs. (9)-(14) under these two conditions, we obtain the following dispersion relation

$\gamma^{5}+a_{4} \gamma^{4}+a_{3} \gamma^{3}+a_{2} \gamma^{2}+a_{1} \gamma+a_{0}=0$,

where

$$
\begin{aligned}
a_{4}= & 2 \omega_{\eta}+2 \omega_{v}+\omega_{\chi}, \\
a_{3}= & \left(\omega_{\eta}+\omega_{v}\right)^{2}+2\left(\omega_{\eta} \omega_{v}+\omega_{\chi} \omega_{\eta}+\omega_{\nu} \omega_{\chi}\right)+2 \omega_{\mathrm{A}}^{2}+\omega_{\mathrm{g}}^{2}+Q^{2}, \\
a_{2}= & \omega_{\chi}\left(\omega_{v}+\omega_{\eta}\right)^{2}+2 \omega_{\eta} \omega_{\nu}\left(\omega_{v}+\omega_{\eta}+\omega_{\chi}\right) \\
& +\omega_{v}\left(\omega_{\mathrm{g}}^{2}+2 \omega_{\mathrm{A}}^{2}\right)+2 \omega_{\eta}\left(\omega_{\mathrm{g}}^{2}+\omega_{\mathrm{A}}^{2}+Q^{2}\right)+\omega_{\chi}\left(Q^{2}+2 \omega_{\mathrm{A}}^{2}\right), \\
a_{1}= & 2\left(\omega_{\nu} \omega_{\eta}+\omega_{\mathrm{A}}^{2}\right)\left(\omega_{\nu} \omega_{\chi}+\omega_{\nu} \omega_{\eta}+\omega_{\eta} \omega_{\chi}\right) \\
& +\omega_{\eta}\left[\left(\omega_{\eta}+2 \omega_{\nu}\right) \omega_{\mathrm{g}}^{2}+\left(\omega_{\eta}+2 \omega_{\chi}\right) Q^{2}-\omega_{\eta} \omega_{v}^{2}\right] \\
& +\omega_{\mathrm{A}}^{2}\left(\omega_{\mathrm{g}}^{2}+\omega_{\mathrm{A}}^{2}+F^{2}\right) \\
a_{0}= & \omega_{\eta}^{2}\left(\omega_{\chi} \omega_{v}^{2}+\omega_{\nu} \omega_{\mathrm{g}}^{2}+\omega_{\chi} Q^{2}\right)+2 \omega_{\mathrm{A}}^{2} \omega_{\nu} \omega_{\eta} \omega_{\chi} \\
& +\omega_{\mathrm{A}}^{2}\left(\omega_{\eta} \omega_{\mathrm{g}}^{2}+\omega_{\chi} \omega_{\mathrm{A}}^{2}+\omega_{\chi} F^{2}\right),
\end{aligned}
$$

and

$$
\begin{aligned}
& Q^{2}=4 \Omega^{2} \frac{k_{z}^{2}}{k^{2}}+F^{2}, \quad F^{2}=\frac{s k_{z}}{k^{2}}\left(k_{z} \Omega_{s}^{2}-k_{s} \Omega_{z}^{2}\right), \\
& \Omega_{s}^{2}=\frac{\partial \Omega^{2}}{\partial s}, \quad \Omega_{z}^{2}=\frac{\partial \Omega^{2}}{\partial z}, \quad \omega_{\mathrm{A}}=\frac{\boldsymbol{k} \cdot \boldsymbol{B}}{\sqrt{4 \pi \rho}}, \\
& \omega_{\mathrm{g}}^{2}=-\boldsymbol{C} \cdot\left[\boldsymbol{G}-\frac{\boldsymbol{k}}{k^{2}}(\boldsymbol{k} \cdot \boldsymbol{G})\right], \quad \boldsymbol{C}=\frac{\beta}{T} \Delta \nabla T .
\end{aligned}
$$

This equation describes five low-frequency modes that exist in differentially rotating and weakly magnetized non-ideal fluids. In the limit of ideal MHD ( $v=\eta=\chi=0)$, Eq. (15) reduces to a quadric one that describes four dynamical modes. The fifth root corresponds to a secular mode that appears only due to dissipative effects, so we address the properties of this mode. In a vanishing magnetic field ( $B=0, \eta=0)$, this mode has been considered by Goldreich \& Schubert (1967) and Fricke (1968). Equation (15) differs only by notations from the dispersion equations derived by Menou et al. (2004). Note, however, that these authors only considered the stability properties of Eq. (15) in a weak magnetic field, $\Omega \gg \omega_{\mathrm{A}}$, and in either the inviscid or perfect-conductor limit. In this paper, the secular stability is considered for a stronger magnetic field with $\omega_{\mathrm{A}}>\Omega$ (but still satisfying the condition $c_{\mathrm{s}}>c_{\mathrm{A}}$ ) and for arbitrary diffusivities.

\section{Criteria of instability}

The Hurwitz theorem presents a sufficient condition of instability and states that Eq. (15) has unstable solutions with a positive real part if one of the following inequalities

$a_{4}<0, \quad a_{0}<0$,

$A_{1} \equiv a_{4} a_{3}-a_{2}<0$,

$A_{2} \equiv a_{2}\left(a_{4} a_{3}-a_{2}\right)-a_{4}\left(a_{4} a_{1}-a_{0}\right)<0$,

$A_{3} \equiv\left(a_{4} a_{1}-a_{0}\right)\left[a_{2}\left(a_{4} a_{3}-a_{2}\right)-a_{4}\left(a_{4} a_{1}-a_{0}\right)\right]$

$$
-a_{0}\left(a_{4} a_{3}-a_{2}\right)^{2}<0
$$

is fulfilled (see Aleksandrov et al. 1985). Since $\omega_{v}, \omega_{\eta}$, and $\omega_{\chi}$ are positively defined quantities, the condition $a_{4}<0$ never applies.

We consider only the criterion $a_{0}<0$ that corresponds to double diffusive instability and is a sufficient condition of this instability, and focus on relatively large wavelengths, $\lambda=2 \pi / k$, for which $\omega_{\mathrm{A}}^{2} \gg \omega_{\nu} \omega_{\eta}$. This condition can be rewritten as

$\lambda>\lambda_{\mathrm{c}}=2 \pi \frac{\sqrt{v \eta}}{c_{\mathrm{A}}}=2 \pi \frac{\sqrt{4 \pi \rho v \eta}}{B_{p}}$,

where $B_{p}=\sqrt{B_{s}^{2}+B_{z}^{2}}$ is the strength of the poloidal field. Note that $\lambda$ should also be small compared to $H$, because we use a local approximation. Following Menou et al. (2004), we can estimate $\rho v \eta \sim(2-6) \times 10^{3} \mathrm{~g} \mathrm{~cm} / \mathrm{s}^{2}$ in the radiative zone. We then have $\lambda_{\mathrm{c}} \sim 10^{3} / B \mathrm{~cm}$, where $B$ is measured in Gauss. This leaves a comfortable range of wavelengths for which $H>\lambda>\lambda_{\mathrm{c}}$ and our local analysis is valid. Note that, although conditions for instability can indeed be found using our approach with accuracy in terms $\sim\left(\lambda_{\mathrm{c}} / \lambda\right)^{2}$, the critical wavelength that discriminates between stability and instability cannot be addressed by this analysis because this wavelength is $\sim \lambda_{\mathrm{c}}$.

Under condition (17), the criterion $a_{0}<0$ reads

$\chi\left(F^{2}+\omega_{\mathrm{A}}^{2}\right)+\eta \omega_{\mathrm{g}}^{2}<0$.

Equation (18) differs from the condition of instability obtained by Fricke (1968) by the last term on the r.h.s. Usually, $\eta / \chi$ is small in stars but, on the contrary, the ratio $\omega_{\mathrm{g}}^{2} / F^{2}$ is large. Therefore, whether or not the contribution of the last term is small will crucially depend on the particular system under consideration. This point concerning viscosity and magnetic diffusivity was first emphasized by Acheson (1978). Condition (18) also differs essentially from the well-known criterion of the magnetorotational instability,

$F^{2}+\omega_{\mathrm{A}}^{2}+\omega_{\mathrm{g}}^{2}<0$

(e.g., Fricke 1969; Balbus \& Hawley 1991; Balbus 1995; Urpin 1996). Note that criterion (19) is valid only if dissipative effects are negligible and the magnetic field is very weak, $\omega_{\mathrm{A}} \ll$ $s\left|\Omega_{s, z}\right|$. Compared to the magnetorotational instability, the stabilizing effect of stratification is much reduced in Eq. (18). Since usually $\omega_{\mathrm{g}}^{2} \gg F^{2}$ in stars, criterion (18) requires a much weaker differential rotation for instability than criterion (19).

In a realistic situation, all three terms in Eq. (18) should be taken into account. Generally, the buoyancy frequency is 
much larger than $\Omega$, and the stratification term is not negligible, even in a fully ionized stellar plasma where $\eta$ is small. The inequality (18) depends on the direction of a wavevector and can be rewritten as follows

$$
\begin{aligned}
N(\boldsymbol{k}) \equiv & F^{2}+\omega_{\mathrm{A}}^{2}+\frac{\eta}{\chi} \omega_{\mathrm{g}}^{2}=\frac{\eta}{\chi} \omega_{0}^{2}+A \frac{k_{z}^{2}}{k^{2}} \\
& -D \frac{k_{s} k_{z}}{k^{2}}+E \frac{k_{s}^{2}}{k^{2}}<0,
\end{aligned}
$$

where

$$
\begin{aligned}
& A=s \Omega_{s}^{2}+\omega_{\mathrm{A} 0}^{2} \cos ^{2} \alpha+\frac{\eta}{\chi} C_{z} G_{z}, \\
& D=s \Omega_{z}^{2}-\omega_{\mathrm{A} 0}^{2} \sin 2 \alpha-\frac{\eta}{\chi}\left(C_{s} G_{z}+C_{z} G_{s}\right), \\
& E=\omega_{\mathrm{A} 0}^{2} \sin ^{2} \alpha+\frac{\eta}{\chi} C_{s} G_{s} .
\end{aligned}
$$

In these expressions, we use notations

$\omega_{0}^{2}=-\boldsymbol{C} \cdot \boldsymbol{G}, \quad \omega_{\mathrm{A} 0}^{2}=\frac{k^{2} B_{p}^{2}}{4 \pi \rho}$,

for the Brunt-Väisälä and Alfven frequences, respectively; $\alpha$ is the angle between the poloidal magnetic field and the axis of rotation. In what follows, we use the procedure proposed by Miralles et al. (2004) to derive the criterion that does not depend on the direction of $\boldsymbol{k}$ and that determines the minimal differential rotation leading to instability. Since the dependence of $N(\boldsymbol{k})$ on the direction of $\boldsymbol{k}$ is rather simple, we obtain that the minimum of $N(\boldsymbol{k})$ corresponds to

$\frac{k_{z}^{2}}{k^{2}}=\frac{1}{2}\left[1 \pm \sqrt{\frac{(A-E)^{2}}{(A-E)^{2}+D^{2}}}\right]$.

Then, the minimum value of $N(\boldsymbol{k})$ yields the following condition of instability

$s \Omega_{s}^{2}+\frac{\eta}{\chi} \omega_{0}^{2}+\omega_{\mathrm{A} 0}^{2} \pm \sqrt{D^{2}+(A-E)^{2}}<0$.

The gradients of $p, \rho$ and $T$ are related by

$$
\frac{\nabla p}{p}=\left(\frac{\partial \ln p}{\partial \ln \rho}\right)_{T}\left(\frac{\nabla \rho}{\rho}+\beta \frac{\nabla T}{T}\right) .
$$

From Eq. (8), we can easily show that the condition of hydrostatic equilibrium leads to

$s \Omega_{z}^{2}=C_{z} G_{s}-C_{s} G_{z}-L_{0 \varphi}$,

where $L_{0 \varphi}=L_{\varphi}-C_{z} G_{B s}+C_{s} G_{B z}$. Then, Eq. (22) can be further simplified to obtain

$$
\begin{aligned}
& s \Omega_{s}^{2}+\frac{\eta}{\chi} \omega_{0}^{2}+\omega_{\mathrm{A} 0}^{2} \pm\left\{\left(s \Omega_{s}^{2}+\frac{\eta}{\chi} \omega_{0}^{2}+\omega_{\mathrm{A} 0}^{2}\right)^{2}\right. \\
& +4 G_{z} \frac{\eta}{\chi}\left[s\left(C_{z} \Omega_{s}^{2}-C_{s} \Omega_{z}^{2}\right)+\omega_{\mathrm{A} 0}^{2}\left(C_{z} \cos 2 \alpha+C_{s} \sin 2 \alpha\right)\right] \\
& -4 \sin ^{2} \alpha \omega_{\mathrm{A} 0}^{2}\left(\frac{\eta}{\chi} \omega_{0}^{2}+s \Omega_{s}^{2}\right)-2\left(1-\frac{\eta}{\chi}\right) s \Omega_{z}^{2} \omega_{\mathrm{A} 0}^{2} \sin 2 \alpha \\
& \left.+\left[\left(1-\frac{\eta}{\chi}\right) s \Omega_{z}^{2}-\frac{\eta}{\chi} L_{0 \varphi}\right]^{2}+2 \frac{\eta}{\chi} L_{0 \varphi} \omega_{\mathrm{A} 0}^{2} \sin 2 \alpha\right\}^{1 / 2}<0 .
\end{aligned}
$$

The two conditions for instability follow straightforwardly from the above expression:

$$
\begin{aligned}
& s \Omega_{s}^{2}+ \frac{\eta}{\chi} \omega_{0}^{2}+\omega_{\mathrm{A} 0}^{2}<0 \\
& 4 G_{z} \frac{\eta}{\chi}\left[s\left(C_{z} \Omega_{s}^{2}-C_{s} \Omega_{z}^{2}\right)+\omega_{\mathrm{A} 0}^{2}\left(C_{z} \cos 2 \alpha+C_{s} \sin 2 \alpha\right)\right] \\
&-4 \sin ^{2} \alpha \omega_{\mathrm{A} 0}^{2}\left(\frac{\eta}{\chi} \omega_{0}^{2}+s \Omega_{s}^{2}\right)-2\left(1-\frac{\eta}{\chi}\right) s \Omega_{z}^{2} \omega_{\mathrm{A} 0}^{2} \sin 2 \alpha \\
&+\left[\left(1-\frac{\eta}{\chi}\right) s \Omega_{z}^{2}-\frac{\eta}{\chi} L_{\varphi}\right]^{2}+2 \frac{\eta}{\chi} L_{\varphi} \omega_{\mathrm{A} 0}^{2} \sin 2 \alpha>0 .
\end{aligned}
$$

These two conditions look like the Solberg-Høiland conditions (Tassoul 2000), but with additional terms due to the magnetic field and diffusive effects. Equations (26) and (27) are equivalent to the condition $a_{0}<0$ with the accuracy in terms $\sim\left(\lambda_{\mathrm{c}} / \lambda\right)^{2}$. Therefore, the boundary between the stable and unstable regions can be determined by these conditions with the same accuracy. If $B=0$ and $\eta=0$, Eq. (27) reduces to the well-known Goldreich-Schubert-Fricke criterion of instability, which states that differential rotation is always unstable if $\Omega_{z}^{2} \neq 0$ (Goldreich \& Schubert 1967; Fricke 1968). Criterion (27) is satisfied in the case of a non-rotating star $(\Omega=0)$ with $B_{\varphi} \gg B_{p}$, when the terms containing $\omega_{\mathrm{A} 0}$ can be neglected, and Eq. (27) yields $\eta^{2} L_{0 \varphi}^{2} / \chi^{2}>0$. Therefore, the star with a presumably toroidal field is always unstable that was first argued by Tayler (1973). Generally, if the poloidal and toroidal fields are more or less comparable, we have $L_{0 \varphi} \sim$ $\omega_{\mathrm{A} 0}^{2}(k H)^{-2} \ll \omega_{\mathrm{A} 0}^{2}$, and the terms proportional to $L_{0 \varphi}$ can be neglected.

\subsection{Instability in a weak magnetic field}

We consider Eqs. (26) and (27) in the limiting cases of weak and relatively strong magnetic fields. We consider the magnetic field to be weak if $\Omega^{2} \gg \omega_{\mathrm{A} 0}^{2}$, assuming that $s \Omega_{s}^{2} \sim s \Omega_{z}^{2} \sim \Omega^{2}$. This condition can be written as

$B_{p}<B_{\mathrm{cr}}=\sqrt{4 \pi \rho} \Omega / k \approx 6 \times 10^{2} \sqrt{\rho} \lambda_{8} \Omega_{-5} \mathrm{G}$

where $\lambda_{8}=\lambda / 10^{8} \mathrm{~cm}, \Omega_{-5}=\Omega / 10^{-5} \mathrm{~s}$, and $\rho$ is measured in $\mathrm{g} / \mathrm{cc}$. The quantity $B_{\mathrm{cr}}$ depends on $\rho$ and, in general, the same field can be considered weak in the inner layers and strong in the outer layers. Note that the field should also satisfy the condition $c_{\mathrm{s}}>c_{\mathrm{A}}$ (Eq. (6)) to be valid in our analysis. It can be convenient to represent the inequality (28) in terms of the equatorial velocity, $V=\Omega R$, where $R$ is the stellar radius. Then, from Eq. (28) we have

$V>17 B_{p 3} R_{11} \lambda_{8}^{-1} \rho^{-1 / 2} \mathrm{~km} \mathrm{~s}^{-1}$,

where $B_{p 3}=B_{p} / 10^{3} \mathrm{G}, R_{11}=R / 10^{11} \mathrm{~cm}$.

In a weak field, Eqs. (26) and (27) yield

$s \Omega_{s}^{2}+\frac{\eta}{\chi} \omega_{0}^{2}<0$

$4 G_{z} \frac{\eta}{\chi} s\left(C_{z} \Omega_{s}^{2}-C_{s} \Omega_{z}^{2}\right)+\left(1-\frac{\eta}{\chi}\right)^{2}\left(s \Omega_{z}^{2}\right)^{2}>0$.

The criteria turn out to be independent of the magnetic field in this limit and, under certain conditions, can be satisfied. 
Since $\omega_{0}^{2}>0$ in the radiative zone, inequality (30) can be satisfied only if $\Omega$ decreases with the cylindrical radius. Taking into account that, typically, $\eta / \chi \sim 10^{-4}$ and $\omega_{0}^{2} \sim 10^{-6}-10^{-7} \mathrm{~s}^{-2}$ in the radiative zone, and assuming that $\left|s \Omega_{s}^{2}\right| \sim \Omega^{2}$, we find that the condition $\eta \omega_{0}^{2} / \chi<\left|s \Omega_{s}^{2}\right|$ is satisfied if $\Omega>10^{-5} \omega_{03} \mathrm{~s}^{-1}$ where $\omega_{03}=\omega_{0} / 10^{-3} \mathrm{~s}$. Therefore, the instability caused by condition (30) may occur in relatively rapidly rotating stars with $\Omega>10^{-5} \omega_{03} \mathrm{~s}^{-1}$ or $V>$ $10 R_{11} \omega_{03} \mathrm{~km} \mathrm{~s}^{-1}$ if $\partial \Omega / \partial s<0$.

Condition (31) depends on the vertical gradient of the angular velocity. If $\Omega_{z}^{2}=0$ then Eq. (31) yields

$4 s \frac{\eta}{\chi} C_{z} G_{z} \Omega_{s}^{2}>0$.

We have $C_{z} G_{z}<0$ since both $\boldsymbol{C}$ and $\boldsymbol{G}$ are approximately radial and go in opposite directions in the radiative zone, if the star is far from the rotational distortion. Therefore, inequality (32) is equivalent to $\Omega_{s}^{2}<0$, and both slowly and rapidly rotating stars can be unstable in this case, if the wavelength of perturbations satisfies condition (17).

If $\Omega_{z}^{2} \neq 0$, then the vertical dependence of the angular velocity can produce either a stabilizing or destabilizing effect depending on $\Omega(z)$. If rotation is slow and $\eta \omega_{0}^{2} / \chi>\Omega^{2}$, then criterion (31) is equivalent to

$G_{z}\left(C_{z} \Omega_{s}^{2}-C_{s} \Omega_{z}^{2}\right)>0$.

In slowly rotating stars, $\boldsymbol{G}$ and $\boldsymbol{C}$ are approximately radial and are directed inward and outward, respectivetely. Then, we have

$G_{z} \approx-G \cos \theta, \quad C_{s} \approx C \sin \theta, \quad C_{z} \approx C \cos \theta$,

where $\theta$ is the polar angle. Substituting these expressions into Eq. (33), we obtain

$\Omega_{s}^{2} \cos ^{2} \theta-\Omega_{z}^{2} \sin \theta \cos \theta<0$.

Hence, $\Omega_{z}^{2}$ provides a destabilizing influence, if $\Omega$ increases with the distance from the equator, and has a stabilizing influence in the opposite case. If $\Omega_{s}^{2}<0$ and $\Omega_{z}^{2}<0$, then instability occurs only in a cone around the rotational axis, and the opening angle of this cone depends on the radial and vertical lengthscale of $\Omega$. On the contrary, if $\Omega_{s}^{2}>0$ and $\Omega_{z}^{2}>0$, then the instability arises only in a region around the equatorial plane.

Rapidly rotating stars with a sufficiently strong vertical differential rotation, $\left|s \Omega_{z}^{2}\right|>\eta \omega_{0}^{2} / \chi$, can be unstable independently of the particular shape of $\Omega(z)$, if $\lambda$ satisfies condition (17); and the instability occurs everywhere within the radiative zone.

\subsection{Instability in a strong magnetic field}

We consider the magnetic field strong if

$B_{p}>B_{\mathrm{cr}}=6 \times 10^{2} \sqrt{\rho} \lambda_{8} \Omega_{-5} \mathrm{G}$

(but condition (6) still holds), because such a field can strongly influence the stability properties. Equation (36) can be rewritten as

$V<17 B_{p 3} R_{11} \lambda_{8}^{-1} \rho^{-1 / 2} \mathrm{~km} \mathrm{~s}^{-1}$.
Under this condition, criteria (26) and (27) transform into

$$
\begin{aligned}
& \frac{\eta}{\chi} \omega_{0}^{2}+\omega_{\mathrm{A} 0}^{2}<0 \\
& 2 G_{z} \frac{\eta}{\chi}\left(C_{z} \cos 2 \alpha+C_{s} \sin 2 \alpha\right)-2 \sin ^{2} \alpha\left(\frac{\eta}{\chi} \omega_{0}^{2}+s \Omega_{s}^{2}\right) \\
& \quad-\left(1-\frac{\eta}{\chi}\right) s \Omega_{z}^{2} \sin 2 \alpha>0 .
\end{aligned}
$$

The first criterion never applies in the radiative zone, since $\omega_{0}^{2}>0$, and we consider only condition (39). Note that this criterion turns out to be independent of the strength of the magnetic field but depends on its direction.

In slowly rotating stars with $\eta \omega_{0}^{2} / \chi>\Omega^{2}$, from Eq. (39) we have

$G_{z}\left(C_{z} \cos 2 \alpha+C_{s} \sin 2 \alpha\right)-\omega_{0}^{2} \sin ^{2} \alpha>0$

or, using Eq. (34),

$(\cos \theta \cos \alpha-\sin \theta \sin \alpha)^{2}<0$,

which never applies. Therefore, the radiative zones of slowly rotating stars with strong magnetic fields satisfying inequality (36) should be secularly stable.

Consider the case of a relatively rapid rotation, $\Omega^{2}>$ $\eta \omega_{0}^{2} / \chi$, or $V>10 R_{11} \omega_{03} \mathrm{~km} \mathrm{~s}^{-1}$. Combining this condition with inequality (37), we obtain a range of $V$ where rotation can be considered rapid and the magnetic field strong

$17 B_{p 3} R_{11} \lambda_{8}^{-1} \rho^{-1 / 2} \mathrm{~km} \mathrm{~s}^{-1}>V>10 R_{11} \omega_{03} \mathrm{~km} \mathrm{~s}^{-1}$.

Equation (39) yields within this range

$\sin \alpha\left(\Omega_{s}^{2} \sin \alpha+\Omega_{z}^{2} \cos \alpha\right)<0$

(we neglect the term $\eta / \chi$ compared to unit). If rotation is cylindrical and $\Omega_{z}^{2}=0$, then instability occurs when the angular velocity decreases with the cylindrical radius.

Stability properties can be more complicated, however, if rotation departs from a simple cylindrical law. Consider, for example, the so-called shellular rotation when $\Omega$ is approximately constant on spherical shells, $\Omega=\Omega(r)$, where $r$ is the spherical radius. Such rotation can be a reasonable approximation, for example, in the radiative zones of massive main sequence stars (see, e.g., Zahn 1992; Urpin et al. 1996). For a shellular rotation, we have $\Omega_{s}^{2}=\Omega_{r}^{2} \sin \theta$ and $\Omega_{z}^{2}=\Omega_{r}^{2} \cos \theta$, where $\Omega_{r}^{2}=\partial \Omega^{2} / \partial r$. Then, condition (43) reads

$\Omega_{r}^{2} \sin \alpha(\sin \alpha \sin \theta+\cos \alpha \cos \theta)<0$.

If the angular velocity decreases with the spherical radius, $\Omega_{r}^{2}<0$, the instability occurs in the region where

$\sin \alpha \cos (\alpha-\theta)>0$.

There is no generally accepted point of view regarding the geometry and strength of the magnetic field inside stars. For the purpose of illustration, we consider, therefore, the simplest magnetic configuration that can exist in the radiative zone

$B_{r}=f(r)\left(1-3 \cos ^{2} \theta\right), \quad B_{\theta}=F(r) \sin \theta \cos \theta$, 
Table 1. The dependence of the region of instability on $q$ for a rapid shellular rotation.

\begin{tabular}{llll}
\hline \hline$q$ & $q>0$ & $0>q>-2$ & $-2>q$ \\
\hline Unstable & $\cos \theta<\sqrt{3} / 3$ & $\cos \theta<\sqrt{3} / 3$ & $\cos \theta<1 / \sqrt{1-q}$ \\
region & & $\cos \theta>1 / \sqrt{1-q}$ & $\cos \theta>\sqrt{3} / 3$ \\
\hline
\end{tabular}

where $f$ and $F$ are functions of the spherical radius alone, and they satisfy the divergence-free condition

$F=\frac{1}{r} \frac{\mathrm{d}\left(r^{2} f\right)}{\mathrm{d} r}$.

The magnetic field (46) has the same geometry as the velocity pattern of meridional circulation caused by shellular rotation with $\Omega=\Omega(r)$ (Zahn 1992; Urpin et al. 1996; Talon et al. 1997). Therefore, assumption (46) is a good approximation if the field is frozen in a slow circulation flow. Generally, the field in radiative zones can have a more complex geometry but simple model (46) allows us to qualitatively understand how the magnetic field influences the region of instability.

We have for magnetic field (46)

$\sin \alpha=\frac{\boldsymbol{e}_{s} \cdot \boldsymbol{B}}{B_{p}}=\frac{\sin \theta}{B_{p}}\left[f\left(1-3 \cos ^{2} \theta\right)+F \cos ^{2} \theta\right]$,

$\cos \alpha=\frac{\boldsymbol{e}_{z} \cdot \boldsymbol{B}}{B_{p}}=\frac{\cos \theta}{B_{p}}\left[f\left(1-3 \cos ^{2} \theta\right)-F \sin ^{2} \theta\right]$.

Substituting these expressions into Eq. (44), we obtain

$\left(1-3 \cos ^{2} \theta\right)\left[1-(1-q) \cos ^{2} \theta\right]>0$,

where $q=\operatorname{dln} f / \operatorname{dln} r$. Criterion (49) is fulfilled at $\cos \theta \approx 0$, and the region near the equator is secularly unstable. Near the rotation axis where $\cos \theta \approx 1$, Eq. (49) yields

$$
\frac{\mathrm{d} f}{\mathrm{~d} r}<0
$$

and this region can be unstable only if the radial field component decreases to the surface. The regions of instability as a function of the parameter $q$ are shown in Table 1 .

There is a principal difference between the cases $q<0$ and $q>0$. The first case can correspond to the field generated somewhere in the central region of the star, such as in the convective core that exists in massive stars. The second case is more likely relevant to the field generated in the surface layers. For instance, this can be typical of the late-type stars that have outer convective zones where a turbulent dynamo operates. If the field increases with $r$ and $q>0$, then the instability occurs only in the region with an opening angle $\approx 36^{\circ}$ around the equator. A wide region around the rotation axis with $\theta<54^{\circ}$ is stable. In contrast, if the field decreases to the surface and $q<0$, then there are two different unstable regions. For a slowly decreasing field, $0>q>-2$, the instability occurs in a relatively wide unstable region around the equator (with the opening angle $\theta>54^{\circ}$ ) and in a narrow region around the axis (with the opening angle $\arccos 1 / \sqrt{1-q}$ ). If the field decreases rapidly with $r$ and $q<-2$, then the instability occurs in a region with $\theta<36^{\circ}$ around the rotation axis and in a narrow region around the equator (with the opening angle $\arccos 1 / \sqrt{1-q}$ ). Note that this opening angle can be very small if the field decreases rapidly.

\section{The growth rate of instability}

We consider the growth rate of the modes assuming that the dissipative frequences $\omega_{\eta}, \omega_{v}$, and $\omega_{\chi}$ are smaller than the dynamical frequences $\omega_{\mathrm{A}}, \omega_{\mathrm{g}}$, and $\Omega$, because this case is of particular interest for stellar radiative zones. Under this assumption, dispersion Eq. (15) can be solved by using the perturbation procedure. We expand $\gamma$ as $\gamma=\gamma^{(0)}+\gamma^{(1)}+\ldots$ where $\gamma^{(0)}$ and $\gamma^{(1)}$ are terms of the zeroth and first order in dissipative frequencies, respectively. The corresponding expansion should be made for the coefficients of Eq. (15) as well. In the zeroth order, when dissipation is neglected, Eq. (15) reduces to a quadratic equation,

$\gamma^{4}+\left(2 \omega_{\mathrm{A}}^{2}+\omega_{\mathrm{g}}^{2}+Q^{2}\right) \gamma^{2}+\omega_{\mathrm{A}}^{2}\left(\omega_{\mathrm{A}}^{2}+\omega_{\mathrm{g}}^{2}+F^{2}\right)=0$.

This equation describes four modes with the frequencies

$$
\begin{aligned}
& \gamma_{1,2}^{(0) 2}=-\omega_{\mathrm{A}}^{2}-\frac{\omega_{\mathrm{g}}^{2}+Q^{2}}{2}+\sqrt{\frac{\left(\omega_{\mathrm{g}}^{2}+Q^{2}\right)^{2}}{4}+4 \omega_{\mathrm{A}}^{2} \Omega^{2} \frac{k_{z}^{2}}{k^{2}}}, \\
& \gamma_{3,4}^{(0) 2}=-\omega_{\mathrm{A}}^{2}-\frac{\omega_{\mathrm{g}}^{2}+Q^{2}}{2}-\sqrt{\frac{\left(\omega_{\mathrm{g}}^{2}+Q^{2}\right)^{2}}{4}+4 \omega_{\mathrm{A}}^{2} \Omega^{2} \frac{k_{z}^{2}}{k^{2}}} .
\end{aligned}
$$

The fifth root of Eq. (15) vanishes in the zeroth approximation, $\gamma_{5}^{(0)}=0$. Equation (51) has unstable solutions with $\operatorname{Re} \gamma>0$, if one of the conditions.

$2 \omega_{\mathrm{A}}^{2}+\omega_{\mathrm{g}}^{2}+Q^{2}<0, \quad \omega_{\mathrm{A}}^{2}+\omega_{\mathrm{g}}^{2}+F^{2}<0$,

is satisfied. The first of these inequalities describes the wellknown Rayleigh instability, and the second condition corresponds to the magnetorotational instability (Velikhov 1959; Chandrasekhar 1960; Balbus \& Hawley 1991). Most likely, neither of inequalities (54) is fulfilled in radiative zones because of strong stabilizing effect of stratification. The only exception are the perturbations with $\boldsymbol{k} \| \boldsymbol{G}$ for which $\omega_{\mathrm{g}}^{2}=0$ (Urpin 1996). Note that the stabilizing effect of stratification is very suppressed for the instability considered in this paper (see Eq. (18)) compared to the Rayleigh and magnetorotational instabilities,

The fifth root of Eq. (15) describes a secular mode which is linear in dissipative effects. To calculate this root we should keep in Eq. (15) only the terms that are linear in dissipative frequencies, assuming that $\gamma_{5}$ is linear as well. Then, the fifth root is approximately given by

$\gamma_{5}^{(1)} \approx-\omega_{\chi} \frac{F^{2}+\omega_{\mathrm{A}}^{2}+(\eta / \chi) \omega_{\mathrm{g}}^{2}}{F^{2}+\omega_{\mathrm{A}}^{2}+\omega_{\mathrm{g}}^{2}}$

The order-of-magnitude estimate of this root reads

$\gamma_{5}^{(1)} \sim \omega_{\chi} \frac{\Omega^{2}}{\omega_{\mathrm{g}}^{2}} \sim 4 \times 10^{-10} \chi_{7} \omega_{03}^{-2} R_{11}^{-2} \lambda_{8}^{-2} V_{7}^{2} \mathrm{~s}^{-1}$, 
where $\chi_{7}=\chi / 10^{7} \mathrm{~cm}^{2} / \mathrm{s}$ and $V_{7}=V / 10^{7} \mathrm{~cm} / \mathrm{s}$. Then, the growth time of instability is

$\tau=1 / \gamma_{5}^{(1)} \sim 10^{2} \chi_{7}^{-1} \omega_{03}^{2} R_{11}^{2} \lambda_{8}^{2} V_{7}^{-2}$ yr.

The growth time can be rather short if the star rotates rapidly: $V_{7} \sim 1$.

\section{Discussion}

We have considered the diffusive instability of differentially rotating magnetized stellar radiative zones. The instability considered here is a generalization of the well-known Goldreich-Schubert-Fricke instability for the case of magnetic stars. In non-magnetic stars, this instability occurs if the angular velocity depends on the vertical coordinate, $\Omega=\Omega(z)$. The magnetic field, however, changes the stability properties of differentially rotating stars drastically. Even in a weak field satisfying inequality (28), the magnetic analogue of the Goldreich-Schubert-Fricke instability may occur in a region where $\Omega$ depends on the cylindrical radius alone, $\Omega=\Omega(s)$. The necessary condition of instability in this case is a decrease of the angular velocity with the cylindrical radius. If $\partial \Omega / \partial s<0$, then the instability may occur in both slowly and rapidly rotating stars. If rotation is more complicated, $\Omega=$ $\Omega(s, z)$, then the vertical dependence of $\Omega$ can provide either a stabilizing or destabilizing effect depending on the value and particular shape of $\Omega(s, z)$. In rapidly rotating stars with the equatorial velocity $V>10 R_{11} \omega_{03} \mathrm{~km} \mathrm{~s}^{-1}$, the vertical dependence of $\Omega$ plays a destabilizing role, and the radiative zones of such stars should be unstable. In slowly rotating stars, the vertical dependence destabilizes differential rotation if $\partial \Omega / \partial z>0$, and stabilizes if $\partial \Omega / \partial z<0$. In such stars, generally, the instability can occur only in some regions near the rotation axis or equator depending on the particular shape of $\Omega(s, z)$.

In a strong magnetic field with $B_{p}>B_{\text {cr }}$ (but still satisfying the condition $c_{\mathrm{s}}>c_{\mathrm{A}}$ ), the stability properties are different. The secular instability does not occur in slowly rotating stars with the equatorial velocity $V<10 R_{11} \omega_{03} \mathrm{~km} \mathrm{~s}^{-1}$. However, if rotation is rapid and satisfies condition (42) then the radiative zone or its fraction can be subject to instability. The geometry of unstable regions is sensitive to the particular shape of $\Omega(s, z)$. Even in our very simplified model where the axisymmetric magnetic field is considered, the instability may occur, not in the whole radiative zone, but only within some regions restricted by cones in the meridional plane. For example, in the case of a shellular rotation, when $\Omega$ depends on the spherical radius alone, the polar angle corresponding to the unstable regions is determined by the radial dependence of the magnetic field and is shown in Table 1. If the radial magnetic field increases to the surface, then the instability arises only in the region around the equator. In the opposite case, when $B_{r}$ decreases to the surface, the instability occurs both around the equator and rotation axis. The opening angle of unstable regions depends on how fast the magnetic field decreases with $r$.

The growth time of instabilities is relatively short and it operates likely in a non-linear regime. We can estimate the saturation velocity using the mixing-length model (e.g., Schwarzschild 1958) that assumes that the turn-over time of turbulence generated by instability is on the order of the growth time of this instability. In a vanishing magnetic field, this simple approach provides a quantitatively correct estimate of the saturation velocity generated by the Goldreich-Schubert-Fricke instability in stellar conditions (Korycansky 1991) and accretion disks (see Urpin 2003c and Arlt \& Urpin 2004). Then, the saturation velocity in a turbulent cell with lengthscale $\lambda$ can be estimated as $v_{\mathrm{T}}(\lambda) \sim \lambda \gamma(\lambda)$ or, using Eq. (57), as

$v_{\mathrm{T}} \sim 0.04 \chi_{7} \omega_{03}^{-2} R_{11}^{-2} \lambda_{8}^{-1} V_{7}^{2} \mathrm{~cm} / \mathrm{s}$.

It is worth noting that $v_{\mathrm{T}}$ is larger for small scale motions.

Turbulent motions can enhance transport and mix the material in the unstable regions. The coefficient of turbulent diffusion, $\nu_{\mathrm{T}}$, can be estimated as a product of the turbulent velocity and lengthscale. From this, we have

$v_{\mathrm{T}} \sim v_{\mathrm{T}} \lambda \sim 4 \times 10^{6} \chi_{7} \omega_{03}^{-2} R_{11}^{-2} V_{7}^{2} \mathrm{~cm}^{2} / \mathrm{s}$.

Turbulent diffusion caused by the instability is sufficient to mix the unstable zone on a relatively short timescale. Using Eq. (59), we can estimate the timescale of mixing as

$\tau_{\text {mix }} \sim \frac{R^{2}}{v_{\mathrm{T}}} \sim 6 \times 10^{7} \chi_{7}^{-1} \omega_{03}^{2} R_{11}^{4} V_{7}^{-2} \mathrm{yr}$,

which can be substantially shorter than the lifetime of many stars. Therefore, elements from a deep interior can very likely be transported to the stellar surface by turbulence within the unstable zones.

This conclusion is particularly important for strongly magnetized stars where the rotation velocity satisfies condition (42). In such stars, the instability leads to formation of nonspherical "turbulent zones" that mix the material of the deep interiors and surface layer. In our very idealized model with axisymmetric magnetic field and shellular rotation, the instability can form two "mixing zones": the cone turbulent region around the rotation axis and the turbulent belt around the equator. If rotation or the magnetic field is more complex, then the geometry of turbulent "mixing zones" will be more complicated. For example, if the magnetic field is non-axisymmetric, then the distribution of unstable regions should be non-axisymmetric as well. The material should be well mixed in the "mixing zones", and most likely, this mixed material can even reach the atmosphere if the unstable region extends to the surface. If the magnetic field is axisymmetric, then the peculiar regions where the "mixing zones" reach the surface, are likely to have the beltlike structure or to form the polar spot with a peculiar composition. If the field is non-axisymmetric, then the instability can lead to formation of the spot-like structure with chemical pecularities.

\section{References}

Acheson, D. 1978, Phil. Trans. R. Soc. London Ser. A, 289, 459

Acheson, D. 1979, Sol. Phys., 62, 23

Acheson, D., \& Gibson, M. 1978, Phil. Trans. R. Soc. London Ser. A, 289,495

Aleksandrov, A., Kolmogorov, A., \& Laurentiev, M. 1985, Mathematics: Its Content, Methods, and Meaning (Cambridge: MIT) 
Arlt, R., \& Urpin, V. 2004, A\&A, 426, 755

Balbus, S. A. 1995, ApJ, 453, 380

Balbus, S. A., \& Hawley, J. F. 1991, ApJ, 376, 214

Chandrasekhar, S. 1960, Proc. Natl. Acad. Sci., 46, 253

Fricke, K. 1968, Z. Astrophys., 68, 317

Fricke, K. 1969, A\&A, 1, 388

Gilman, P. 1970, ApJ, 162, 1019

Goldreich, P., \& Schubert, G. 1967, ApJ, 150, 571

Goossens, M., \& Tayler, R. J. 1980, MNRAS, 193, 833

Knobloch, E., \& Spruit, H. 1982, A\&A, 113, 261

Korycansky, D. 1991, ApJ, 381, 515

Landau, L., \& Lifshitz, E. 1981, Fluid Mechanics (London: Pergamon Press)

Menou, K. 2004, MNRAS, 352, 1381

Menou, K., Balbus, S., \& Spruit, H. 2004, ApJ, 607, 564
Miralles, J., Pons, J., \& Urpin, V. 2004, A\&A, 420, 245

Schmitt, J., \& Rosner, R. 1983, ApJ, 265, 901

Schwarzschild, M. 1958, Structure and Evolution of the Stars (Princeton: Princeton Univ. Press)

Talon, S., Zahn, J.-P., Maeder, A., \& Meynet, G. 1997, A\&A, 322, 209

Tassoul, J.-L. 2000, Stellar Rotation (Cambridge: Cambridge Univ. Press)

Tayler, R. J. 1973, MNRAS, 161, 365

Urpin, V. 1996, MNRAS, 280, 149

Urpin, V. 2003a, A\&A, 408, 331

Urpin, V. 2003b, A\&A, 410, 975

Urpin, V. 2003c, A\&A, 404, 397

Urpin, V., Shalybkov, D., \& Spruit, H. 1996, A\&A, 306, 455

Velikhov, E. P. 1959, Sov. Phys. JETP, 9, 995

Zahn, J.-P. 1992, A\&A, 265, 115 